\title{
Transient dynamics and waiting time distribution of molecular junctions in the polaronic regime
}

\author{
R. Seoane Souto, ${ }^{1}$ R. Avriller, ${ }^{2}$ R. C. Monreal, ${ }^{1}$ A. Martín-Rodero, ${ }^{1}$ and A. Levy Yeyati ${ }^{1}$ \\ ${ }^{1}$ Departamento de Física Teórica de la Materia Condensada, Condensed Matter Physics Center (IFIMAC) and Instituto Nicolás Cabrera, \\ Universidad Autónoma de Madrid, E-28049 Madrid, Spain \\ ${ }^{2}$ Université de Bordeaux, LOMA, UMR 5798, F-33400 Talence, France \\ and CNRS, LOMA, UMR 5798, F-33400 Talence, France
}

(Received 9 July 2015; revised manuscript received 4 September 2015; published 25 September 2015)

\begin{abstract}
We develop a theoretical approach to study the transient dynamics and the time-dependent statistics for the Anderson-Holstein model in the regime of strong electron-phonon coupling. For this purpose we adapt a recently introduced diagrammatic approach to the time domain. The generating function for the time-dependent charge transfer probabilities is evaluated numerically by discretizing the Keldysh contour. The method allows us to analyze the system evolution to the steady state after a sudden connection of the dot to the leads, starting from different initial conditions. Simple analytical results are obtained in the regime of very short times. We study in particular the apparent bistable behavior occurring for strong electron-phonon coupling, small bias voltages, and a detuned dot level. The results obtained are in remarkably good agreement with numerically exact results obtained by quantum Monte Carlo methods. We analyze the waiting time distribution and charge transfer probabilities, showing that only a single electron transfer is responsible for the rich structure found in the short-time regime. A universal scaling (independent of the model parameters) is found for the relative amplitude of the higher order current cumulants in the short-time regime, starting from an initially empty dot. We finally analyze the convergence to the steady state of the differential conductance and of the differential Fano factor at the inelastic threshold, which exhibits a peculiar oscillatory behavior.
\end{abstract}

PACS number(s): 72.70.+m, 73.63.-b, 73.23.-b, 85.65.+h

\section{INTRODUCTION}

The study of time-dependent current fluctuations in nanoscale conductors is of great importance as it can provide information on the interactions and quantum correlations between electrons [1,2]. While these studies have been traditionally restricted to the stationary regime (corresponding to long measuring times), the advent of single-electron sources [3-5] has triggered the interest in the short-time behavior. On the one hand this knowledge would be useful to fully characterize the single-electron emitters in the high-frequency range [6]. On the other hand, understanding the short-time dynamics is a necessary requirement for the use of nanodevices in the detection of individual electrons [7].

This context suggests the need of developing new methods to characterize the statistics in the time domain. The concept of waiting time distributions (WTDs) is well known in the field of quantum optics and stochastic processes [8] but has been more recently introduced in electronic transport [9]. Here it has been studied in the incoherent regime using master equations both within Markovian [9-11] and non-Markovian [12] approximations. The extension of these studies to the coherent (but noninteracting) regime is a quite recent development. For this case a scattering approach has been introduced [13] and adapted later to tight-binding models [14]. Another approach to the problem is provided by nonequilibrium Green's function methods, which have been discussed in Ref. [15] and applied to analyze the transient dynamics of noninteracting quantum dots in Refs. [16,17].

Green's function methods are in principle the most appropriate to study the effect of interactions in the time-dependent statistical properties of quantum coherent conductors. However, their application to this case has remained essentially unexplored. In the present work we provide some initial steps in this direction by analyzing the Anderson-Holstein model in the polaronic regime. This simple model provides the basis to understand quite complex nonequilibrium phenomena occurring in actual systems such as phonon-assisted tunneling [18,19] and Franck-Condon blockade [20].

While the stationary transport properties of the AndersonHolstein model have been extensively analyzed [21-26], their analysis in the time domain has been much less analyzed [27]. Recent calculations, based on numerically exact methods such as diagrammatic quantum Monte Carlo (diagMC) [28], have indicated that for strong electron-phonon coupling there exists a regime in which different initial conditions lead to different transport properties at short times. In a subsequent work [29] it was demonstrated that this apparent bistability actually corresponds to a long transient dynamics leading to blockingdeblocking events associated with the polaron dynamics. More recent work [30] has confirmed the analysis and also explored the effect of including the dot-leads Coulomb repulsion. The approach to the steady state has also been analyzed for the Anderson-Holstein model including a continuous distribution of phonon modes [31].

In spite of these efforts, none of these works have analyzed the time evolution of the noise properties of the AndersonHolstein model as the system approaches the steady state. This deficit connects with the above-mentioned lack of studies of time-dependent statistics for interacting systems in the quantum transport regime. Unfortunately, numerically exact methods such as quantum Monte Carlo [32,33] or numerical renormalization group (NRG) [34] have not yet been adapted to noise studies.

To circumvent these difficulties, the present work introduces a generalization to the time-dependent case of a simple analytical approach called the dressed tunneling approximation (DTA), which was shown to give a good description of 
the spectral and transport properties in the stationary limit for the polaronic regime [35]. As a first step we check that the method provides results for the time-evolution of the mean current and the dot charge which are in good agreement with numerically exact results of Ref. [28]. We then study the transient WTD and the evolution of the current cumulants, showing that interactions tend to increase the characteristic times for relaxation towards the steady state and also enhance the asymmetry in the charge transfer probability distributions. We also study the scaling of the relative amplitudes of the transient cumulants of higher order and find a very robust universal behavior, which we demonstrate using analytical arguments. Finally, we analyze the convergence to the steady state of the differential conductance and of the differential Fano factors at the inelastic threshold $V=\omega_{0}$ and also at $V=2 \omega_{0}$.

\section{MODEL AND BASIC THEORETICAL FORMULATION}

We consider the spinless Anderson-Holstein model in which a single electronic level is coupled to a localized vibrational mode. Electrons can tunnel from this resonant level into a left (L) and a right electrode (R). We shall generically refer to this central region, which can represent either a molecule, an atomic chain, or a quantum dot, as the "dot" region. The corresponding Hamiltonian is given by $H=H_{\text {leads }}+H_{\text {dot }}+H_{T}$, with (in natural units, $\hbar=k_{B}=$ $\left.e=m_{e}=1\right)$

$$
H_{\mathrm{dot}}=\left[\epsilon_{0}+\lambda\left(a^{\dagger}+a\right)\right] d^{\dagger} d+\omega_{0} a^{\dagger} a,
$$

where $\epsilon_{0}$ is the bare electronic level, $\lambda$ is the electron-phonon coupling constant, and $\omega_{0}$ is the frequency of the localized vibration. The electron (phonon) creation operator in the dot is denoted by $d^{\dagger}\left(a^{\dagger}\right)$. On the other hand, $H_{\text {leads }}=\sum_{v k} \epsilon_{v k} c_{\nu k}^{\dagger} c_{\nu k}$ corresponds to the noninteracting-leads Hamiltonian $(v \equiv$ $L, R)$ where $\epsilon_{v k}$ are the leads' electron energies and $c_{\nu k}^{\dagger}$ are the corresponding creation operators. The bias voltage applied to the junction is imposed by shifting symmetrically the chemical potential of the electrodes $V=\mu_{L}-\mu_{R}$.

The tunneling processes are described by

$$
H_{T}=\sum_{\nu k}\left(\gamma_{\nu k} c_{\nu k}^{\dagger} d+\text { H.c. }\right),
$$

where $\gamma_{v k}$ are the tunneling amplitudes.

To address the polaronic regime we perform the so-called Lang-Firsov unitary transformation [36] which eliminates the linear term in the electron-phonon coupling [37]:

$$
\bar{H}=S H S^{\dagger}, \quad S=e^{g d^{\dagger} d\left(a^{\dagger}-a\right)}, \quad g=\frac{\lambda}{\omega_{0}} .
$$

Using this transformation

$$
\bar{H}_{\mathrm{dot}}=\tilde{\epsilon} d^{\dagger} d+\omega_{0} a^{\dagger} a,
$$

where $\tilde{\epsilon}=\epsilon_{0}-\lambda^{2} / \omega_{0}$, while the tunneling Hamiltonian is transformed as

$$
\bar{H}_{T}=\sum_{\nu k}\left(\gamma_{\nu k} c_{\nu k}^{\dagger} X d+\text { H.c. }\right)
$$

where $X=\exp \left[g\left(a-a^{\dagger}\right)\right]$ is the phonon cloud operator. On the other hand, the free-leads Hamiltonian remains invariant. For later use it is useful to introduce the tunneling rates
$\Gamma_{v}=\operatorname{Im} \sum_{k}\left|\gamma_{\nu k}\right|^{2} /\left(\omega-i 0^{+}-\epsilon_{\nu k}\right)$ which are approximated by constants in the so-called wide-band approximation, and denote $\Gamma=\Gamma_{L}+\Gamma_{R}$.

In the present work we focus on the transient dynamics which corresponds to the evolution of the system from an initial $t=0$ state when the dot is suddenly connected to both leads. The corresponding statistical properties of the transferred charges can be obtained from the generating function (GF)

$$
Z(\chi, t)=\sum_{q=-\infty}^{\infty} e^{i q \chi} P_{q}(t),
$$

where $P_{q}(t)$ denotes the probability of transferring $q$ charges through the dot in the measuring time $t$. The GF is in turn related to the cumulant generating function (CGF) by $S(\chi, t)=\ln Z(\chi, t)$, which generates the time-dependent charge cumulants $\left.C_{k}(t)=(-i)^{k} \partial^{k} S / \partial \chi^{k}\right\rfloor_{\chi=0}$. We further define $\left\langle\left\langle I^{k}(t)\right\rangle\right\rangle=\partial C_{k}(t) / \partial t$, which tend to the zero-frequency steady state current cumulants when $t \rightarrow \infty$. Another quantity of interest to characterize the transient statistics is the waiting time distribution $W(t)$. This can be related to the so-called idle-time probability $\Pi(t)=P_{0}(t)$, defined as [8]

$$
\Pi(t)=\int_{0}^{2 \pi} \frac{d \chi}{2 \pi} Z(\chi, t) .
$$

While in a stationary situation the definition of the WTD requires a two-time measurement [13], in the transient case the initial time is fixed at $t=0$ and one can define a single-time measurement WTD as $[8,16]$

$$
W(t)=-\frac{d \Pi(t)}{d t},
$$

which gives the probability that the first electron is detected at a certain time $t$ after the connection to the leads.

The GF can be written [15] as an average of the evolution operator over the Keldysh contour, shown in Fig. 1,

$$
Z(\chi, t)=\left\langle T_{\mathcal{C}} \exp \left\{-i \int_{\mathcal{C}} \bar{H}_{\chi}\left(t^{\prime}\right) d t^{\prime}\right\}\right\rangle,
$$

where $\bar{H}_{\chi}$ is the system Hamiltonian with a counting field $\chi(t)$ which takes the values $\pm \chi$ on the two branches of the Keldysh contour entering as a phase factor modulating the tunnel Hamiltonian, i.e.,

$$
\bar{H}_{T, \chi}=\sum_{\nu k}\left(e^{i \chi_{v} / 2} \gamma_{\nu k} c_{\nu k}^{\dagger} X d+\text { H.c. }\right) .
$$

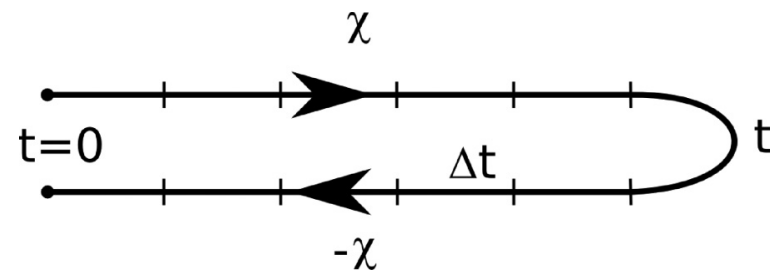

FIG. 1. Keldysh contour considered to analyze the transient regime. $\chi$ indicates the counting field changing sign on the two branches of the contour and $\Delta t$ corresponds to the time step in the discretized calculation of the generating function $Z(\chi, t)$. 
Notice that different charge and current cumulants can be defined depending on how the phase $\chi(t)$ is distributed on the left and on the right tunnel couplings. For instance, taking $\chi_{L}=\chi(t)$ and $\chi_{R}=0, Z(\chi, t)$ generates the current and charge transfer cumulants through the interface between the left lead and the dot. This is the choice that we shall select for the rest of the paper, unless specified explicitly.

\section{A. Noninteracting case}

In Refs. [15,38] it has been shown by path-integral methods that in the noninteracting case $Z(\chi, t)$ can be expressed as the following Fredholm determinant, defined on the Keldysh contour

$$
Z(\chi, t)=\operatorname{det}\left(G \tilde{G}^{-1}\right)=\operatorname{det}\left[G\left(g_{0}^{-1}-\tilde{\Sigma}\right)\right],
$$

where $\tilde{G}$ and $G$ denote the dot Keldysh Green's functions, $g_{0}$ corresponds to the uncoupled dot case, and $\tilde{\Sigma}$ are the selfenergies due to the coupling to the leads. In the quantities $\tilde{G}$ and $\tilde{\Sigma}$ the tilde indicates the inclusion of the counting field in the tunnel amplitudes.

As shown in [39] a simple discretized version of the inverse free dot Green's function on the Keldysh contour is

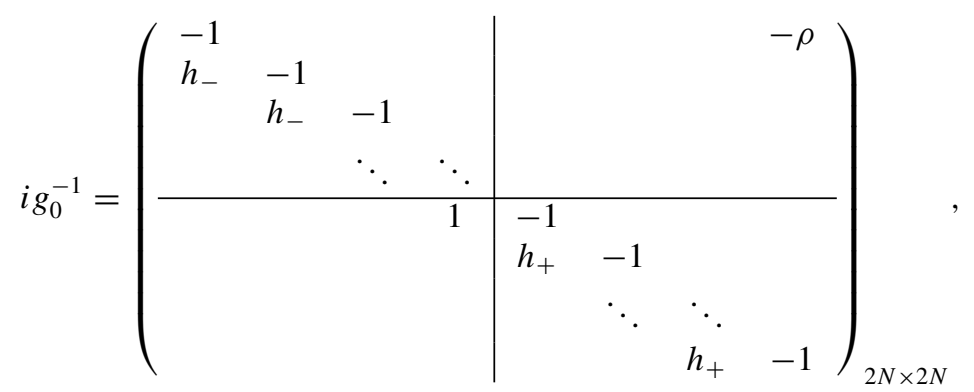

where $h_{ \pm}=1 \mp i \epsilon_{0} \Delta t ; \Delta t$ indicates the time step in the discretization with $N=t / \Delta t$. In this expression $\rho$ determines the initial dot charge $n_{d}$ by $n_{d}=\rho /(1+\rho)$.

On the other hand, the self-energies are given by

$$
\tilde{\Sigma}^{\alpha \beta}\left(t, t^{\prime}\right)=\alpha \beta \theta(t) \theta\left(t^{\prime}\right) \sum_{\nu k} \gamma_{\nu k}^{2} e^{i(\alpha-\beta) \chi_{v} / 2} g_{\nu k}^{\alpha \beta}\left(t, t^{\prime}\right),
$$

where $g_{v k}^{\alpha \beta}\left(t, t^{\prime}\right)=-i\left\langle T_{\mathcal{C}} c_{\nu k}\left(t_{\alpha}\right) c_{\nu k}^{\dagger}\left(t_{\beta}^{\prime}\right)\right\rangle$, with $\alpha, \beta \equiv+,-$ are the Keldysh Green's functions of the uncoupled leads. In Appendix A we discuss the discretization procedure and give the explicit expressions for these self-energies in the discretized contour.

It should be noticed that while $\tilde{\Sigma}$ are $2 \times 2$ block Toeplitz matrices depending only on the time arguments difference, $g_{0}^{-1}$ deviates from a perfect block Toeplitz matrix due to the $(N+1, N)$ and $(1,2 N)$ entries associated with the closing of the Keldysh contour and the initial condition, respectively. The connection with the theory of Toeplitz determinants is an interesting issue [40] that goes beyond the scope of the present work and will be discussed elsewhere.

\section{B. Interacting case: Dressed tunneling approximation}

We now discuss the generalization of the theory to the interacting case within the approximation introduced in in Ref. [35]. For this purpose we start from the counting field functional derivative of the GF:

$$
\begin{aligned}
\frac{\delta Z}{\delta \chi}= & \int_{\mathcal{C}} d t_{1} \sum_{k} \gamma_{L k}\left\langleT _ { \mathcal { C } } \left[ X\left(t_{1}\right) e^{i \chi\left(t_{1}\right) / 2} c_{L k}^{\dagger}\left(t_{1}\right) d\left(t_{1}\right)\right.\right. \\
& \left.\left.-X^{\dagger}\left(t_{1}\right) e^{-i \chi\left(t_{1}\right) / 2} c_{L k}\left(t_{1}\right) d\left(t_{1}\right)\right]\right\rangle .
\end{aligned}
$$

This can be related to the three-point Green's function $\left\langle T_{\mathcal{C}} X(t) c_{L k}^{\dagger}\left(t_{1}\right) d\left(t^{\prime}\right)\right\rangle$, whose equation of motion is

$$
\begin{aligned}
& \left(i \partial_{t_{1}}-\epsilon_{L k}\right)\left\langle T_{\mathcal{C}} X(t) c_{L k}^{\dagger}\left(t_{1}\right) d\left(t^{\prime}\right)\right\rangle \\
& \quad=\gamma_{L k} e^{-i \chi\left(t_{1}\right) / 2}\left\langle T_{\mathcal{C}} X(t) X^{\dagger}\left(t_{1}\right) d^{\dagger}\left(t_{1}\right) d\left(t^{\prime}\right)\right\rangle,
\end{aligned}
$$

which can be integrated yielding

$$
\begin{aligned}
& \left\langle T_{\mathcal{C}} X(t) c_{L k}^{\dagger}\left(t_{1}\right) d\left(t^{\prime}\right)\right\rangle \\
& \quad=\gamma_{L k} \int_{\mathcal{C}} d t_{2} e^{-i \chi\left(t_{1}\right) / 2} g_{L k}\left(t_{2}, t_{1}\right)\left\langle T_{\mathcal{C}} X(t) X^{\dagger}\left(t_{2}\right) d^{\dagger}\left(t_{1}\right) d\left(t^{\prime}\right)\right\rangle .
\end{aligned}
$$

Within the decoupling procedure corresponding to the DTA one has $\left\langle T_{\mathcal{C}} X(t) X^{\dagger}\left(t_{2}\right) d^{\dagger}\left(t_{1}\right) d\left(t^{\prime}\right)\right\rangle \simeq$ $\left\langle T_{\mathcal{C}} X(t) X^{\dagger}\left(t_{2}\right)\right\rangle\left\langle T_{\mathcal{C}} d^{\dagger}\left(t_{1}\right) d\left(t^{\prime}\right)\right\rangle$ which finally allows us to write

$$
\frac{\partial Z}{\partial \chi}=-\int_{0}^{t} d t_{1} \int_{0}^{t} d t_{2} \operatorname{Tr}_{K}\left\{\frac{\partial \tilde{\Sigma}_{\mathrm{DTA}}}{\partial \chi}\left(t_{1}, t_{2}\right) \tilde{G}\left(t_{2}, t_{1}\right)\right\},
$$

where $\operatorname{Tr}_{K}$ denotes trace over the $2 \times 2$ Keldysh space and $\tilde{\Sigma}_{\text {DTA }}$ is the DTA self-energy whose components are given by

$$
\tilde{\Sigma}_{\mathrm{DTA}}^{\alpha \beta}\left(t, t^{\prime}\right)=\tilde{\Sigma}^{\alpha \beta}\left(t, t^{\prime}\right) \Lambda^{\alpha \beta}\left(t, t^{\prime}\right),
$$

with $\Lambda^{\alpha \beta}\left(t, t^{\prime}\right)=\left\langle T_{\mathcal{C}} X(t) X^{\dagger}\left(t^{\prime}\right)\right\rangle$ being the phonon cloud propagator. In this expression $\tilde{\Sigma}^{\alpha \beta}$ denote the self-energies in the noninteracting case given by Eq. (13). On the other hand, the propagator $\Lambda^{\alpha \beta}\left(t, t^{\prime}\right)$ will be evaluated assuming equilibrated phonons (see Appendix B).

Integrating Eq. (17) and imposing the condition $Z(0, t)=1$ one arrives at the same expression for $Z(\chi, t)$ as in Eq. (11) but replacing $\tilde{\Sigma}$ and $G$ by $\tilde{\Sigma}_{\text {DTA }}$ and $G_{\text {DTA }}$. All the effects of interactions are thus encoded in the DTA self-energy $\tilde{\Sigma}_{\text {DTA }}$. More details on the approximation are given in Appendices B and C. It should be noted that this simple structure is 
valid within DTA but in a more general approximation vertex corrections would prevent the counting field integration leading to Eq. (11).

\section{Tunnel and short-time limits}

To the lowest order in $\Gamma$ we have the expansion

$$
\begin{aligned}
Z(\chi, t) \simeq & 1+\int_{0}^{t} d t_{1} \int_{0}^{t} d t_{2} \operatorname{Tr}_{K}\left\{\left[\tilde{\Sigma}_{\mathrm{DTA}}\left(t_{1}, t_{2}\right)\right.\right. \\
& \left.\left.-\Sigma_{\mathrm{DTA}}\left(t_{1}, t_{2}\right)\right] g_{0}\left(t_{2}, t_{1}\right)\right\},
\end{aligned}
$$

which reduces to

$$
\begin{aligned}
Z(\chi, t) \simeq & 1+\int_{0}^{t} d t_{1} \int_{0}^{t} d t_{2}\left[\Sigma_{L, \mathrm{DTA}}^{+-}\left(t_{1}, t_{2}\right) g_{0}^{-+}\left(t_{2}, t_{1}\right)\left(e^{i \chi}-1\right)\right. \\
& \left.+\Sigma_{L, \mathrm{DTA}}^{-+}\left(t_{1}, t_{2}\right) g_{0}^{+-}\left(t_{2}, t_{1}\right)\left(e^{-i \chi}-1\right)\right] .
\end{aligned}
$$

It is interesting to notice that the $\Gamma \rightarrow 0$ and the $t \rightarrow 0$ limits should coincide; i.e., the short-time behavior is well described by the expansion to the lowest order in $\Gamma t$. This allows us to obtain the short-time limit of the GF as

$$
\begin{aligned}
Z(\chi, t) \simeq & 1+\left\{\left(e^{i \chi}-1\right) A_{L 01}(t)\left[1-n_{d}\right]\right. \\
& \left.+\left(e^{-i \chi}-1\right) A_{L 10}(t) n_{d}\right\}
\end{aligned}
$$

with

$$
\begin{aligned}
A_{L 01}(t)= & \frac{2 \Gamma_{L}}{\pi} \\
& \times \sum_{n=0}^{\infty} \alpha_{n} \int_{-W}^{W} d \omega \frac{1-\cos \left[\left(\omega-\tilde{\epsilon}-n \omega_{0}\right) t\right]}{\left(\omega-\tilde{\epsilon}-n \omega_{0}\right)^{2}} f_{L}(\omega), \\
A_{L 10}(t)= & \frac{2 \Gamma_{L}}{\pi} \\
& \times \sum_{n=0}^{\infty} \alpha_{n} \int_{-W}^{W} d \omega \frac{1-\cos \left[\left(\omega-\tilde{\epsilon}+n \omega_{0}\right) t\right]}{\left(\omega-\tilde{\epsilon}+n \omega_{0}\right)^{2}} \\
& \times\left[f_{L}(\omega)-1\right],
\end{aligned}
$$

where $f_{L}(\omega)$ is the Fermi distribution at the left electrode and, at zero temperature, $\alpha_{n}=e^{-g^{2}} g^{2 n} / n !$. The physical interpretation of the amplitudes $A_{L 01}$ and $A_{L 10}$ is transparent in the $t \rightarrow \infty$ limit where $A_{L 01} / t$ and $A_{L 01} / t$ tend to the Fermi golden rule rates derived for the sequential tunneling regime (see, e.g., Ref. [41]).

As expected in the short-time limit $\Gamma t \ll 1$, the GF in Eq. (21) involves charge transfer of a single-electron, with only $P_{0}(t), P_{-1}(t)$, and $P_{1}(t)$ having a significant weight, and are given by

$$
P_{q}(t)=A_{L 01}(t)\left[1-n_{d}\right] \delta_{q, 1}-A_{L 10}(t) n_{d} \delta_{q,-1},
$$

and $P_{0}(t)=1-P_{1}(t)-P_{-1}(t)$. Correspondingly, the WTD is proportional to the left current $I_{L}(t)$, i.e.,

$$
W(t)=-\frac{d}{d t} P_{0}(t)=\left|\frac{I_{L}(t)}{e}\right| .
$$

At zero temperature and neglecting contributions from the band edges (which is justified in the wide band approximation) we obtain

$$
\begin{aligned}
I_{L}= & \Gamma_{L}\left(1-2 n_{d}\right)+\frac{2 \Gamma_{L}}{\pi} \sum_{n} \alpha_{n}\left\{\operatorname{Si}\left[\left(\mu_{L}+n \omega_{0}-\tilde{\epsilon}\right) t\right]\left(1-n_{d}\right)\right. \\
& \left.-\operatorname{Si}\left[\left(\mu_{L}-n \omega_{0}-\tilde{\epsilon}\right) t\right] n_{d}\right\},
\end{aligned}
$$

where Si denotes the sine integral function.

Due to the property $\lim _{x \rightarrow 0} \operatorname{Si}(x)=0$ we find that the initial current is $I_{L}= \pm \Gamma_{L}$, with the sign depending on the initial charge $\left(n_{d}=0\right.$ or $\left.n_{d}=1\right)$. This property fixes also the initial value of the WTD as $W(t) \equiv \operatorname{abs}\left[I_{L}(0)\right]$. It should be noticed that we are neglecting the system evolution on time scales smaller than the inverse of the leads' bandwidth (see Appendix A), which explains why the initial current can be nonzero. However, for the symmetrized current $I(t)=$ $\left[\Gamma_{R} I_{L}(t)-\Gamma_{L} I_{R}(t)\right] / \Gamma$ the initial value is zero and the first charge transfer cumulant is a continuous function starting from zero regardless of the initial condition.

\section{RESULTS}

\section{A. Evolution of mean current and charge: comparison to diagMC results}

We start by analyzing the transient behavior of the Holstein model for different initial conditions. In Ref. [28] it was shown that for certain parameter ranges the model exhibits an apparent bistable behavior. Further analysis [29,30] revealed that this apparent bistability was caused by a long transient regime associated with the slow polaronic dynamics. The comparison of the symmetrized current and the charge evolution obtained within DTA with the numerically exact results obtained by diagMC is shown in Figs. 2 and 3. These results correspond to the case of a deep level $\tilde{\epsilon}=-10 \Gamma$ and large phonon frequency $\omega_{0}=8 \Gamma$ where the apparent bistable behavior is more pronounced. There is a remarkable agreement between the DTA and the diagMC results for the current in the initially

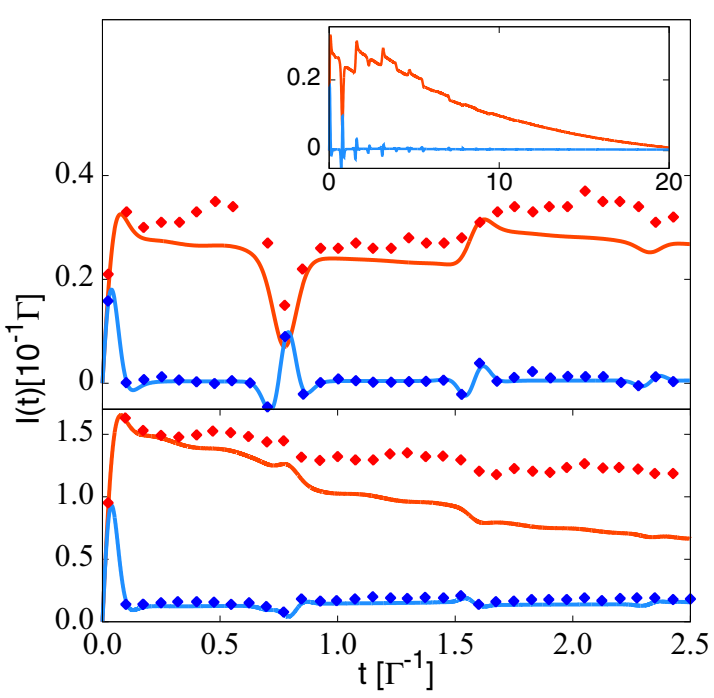

FIG. 2. (Color online) Symmetrized transient current $I(t)$ : comparison between DTA (full lines) and diagMC (dots) for initially empty (red) and initially full (blue) dot. $\tilde{\epsilon}=-10 \Gamma, g=2, \omega_{0}=8 \Gamma$, and $V=5 \Gamma$ (upper panel) and $V=26 \Gamma$ (lower panel). The inset illustrates the convergence to the steady state for the case $V=5 \Gamma$. 


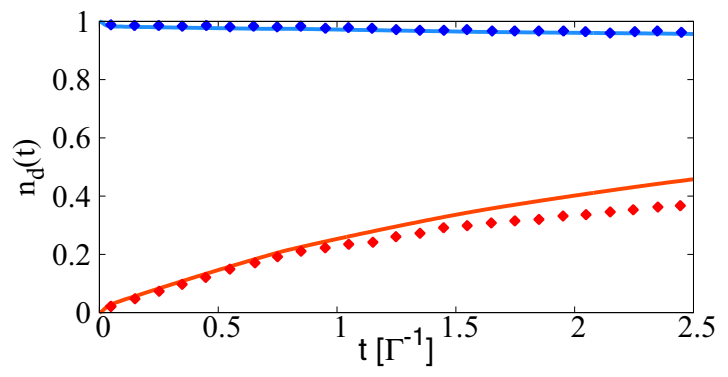

FIG. 3. (Color online) Time-dependent dot occupation: comparison between DTA and diagMC for initially empty and initially full dot. Same parameters as in Fig. 2 (lower panel).

full case. The agreement with the current is somewhat worse for the initially empty case, specially for the larger bias case $V=26 \Gamma$. This reflects a limitation of the DTA for describing the spectral density around the Fermi energy in this regime (see Ref. [35]) although there is a very good agreement in the evolution of the mean charge for this case (see Fig. 3), which represents an improvement with respect to the approximation used in Ref. [29].

The apparent bistable behavior at short times corresponds actually to a long transient dynamics, as illustrated by the DTA results on a longer time scale (see inset in Fig. 2). The results in Fig. 2 also indicate that the typical transient times are reduced with increasing bias voltage. An estimate of this characteristic time can be obtained from the relaxation rate of the retarded Green's function, as given by Eq. (C3), i.e., $\tau \simeq-\operatorname{Re}(1 / r)$. A plot of $\tau$ as function of the interaction strength, $g$, for different bias voltages is included in the Supplemental Material [42]. This characteristic time exhibits a roughly exponential increase with interactions, i.e., $\tau \propto \exp \left[\kappa g^{2}\right]$ (see [42]).

\section{B. Waiting time distribution and transient statistics}

Further insight on the transient dynamics is provided by analyzing the evolution of the WTD and the higher current cumulants; see Fig. 4.

As can be observed, in the initially empty case the WTD exhibits a nonmonotonic decrease with small steps at time $\sim n 2 \pi / \omega_{0}$ associated with the polaron dynamics. The current cumulants in this short-time regime have an increasing amplitude with increasing cumulant order (we come back to this point below). The inset in the upper panel of Fig. 4 indicates that this short-time dynamics is associated with a single electron transfer, with only $P_{0}$ and $P_{1}$ being nonnegligible. Thus the waiting time distribution follows essentially the current at short times. On the other hand, for the initially occupied state the current dynamics is almost blocked in this short-time regime.

As commented on above, the typical times for the transient regime are increased by the effect of interactions. A more clear picture of this effect is provided by Fig. 5 where $W(t)$ is shown for increasing values of $g$ for the initially empty and initially occupied states. To illustrate this effect we have chosen less extreme parameter values than in Figs. 2, 3, 4, for which the apparent bistability is less pronounced.

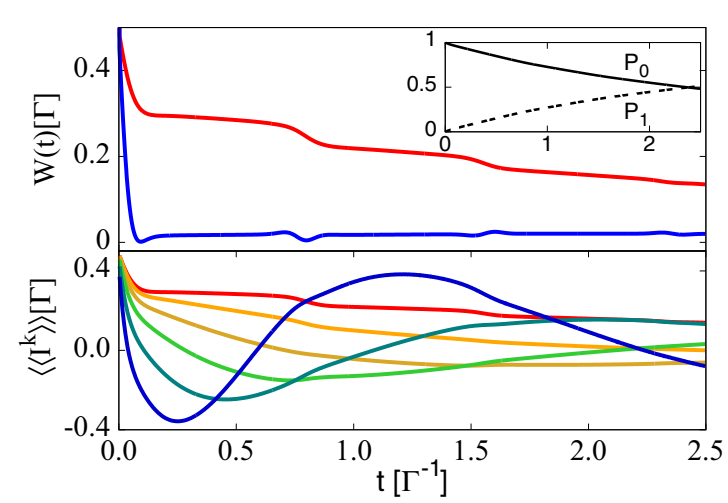

FIG. 4. (Color online) Upper panel: waiting time distribution for the initially empty (red) and initially occupied (blue) cases for the same parameters as in Fig. 2 (lower panel). Inset: probabilities $P_{0}$ (full line) and $P_{1}$ (dashed line) for the initially empty state. The lower panel shows the transient dynamics of current cumulants $\left\langle\left\langle I^{k}(t)\right\rangle\right\rangle$ with $k=1,2,3,4,5,6$ (from top to bottom at short times) for the initially empty case.

At short time scales $\left(t \leqslant \Gamma^{-1}\right)$ and for weak coupling to the leads, in the initially empty case, the WTD is well approximated by Eq. (24). Thus, it exhibits an initial linear behavior fixed by $W(t) \approx \Gamma_{L} / 2+2 \Gamma_{L} / \pi \sum_{n} \alpha_{n}\left(\mu_{L}-\tilde{\epsilon}-\right.$ $\left.n \omega_{0}\right) t$ followed by an extremum at $t \approx \pi\left\{\mid \sum_{n}^{n} \alpha_{n}\left(\mu_{L}-\tilde{\epsilon}-\right.\right.$ $\left.\left.n \omega_{0}\right) / \sum_{n} \alpha_{n}\left(\mu_{L}-\tilde{\epsilon}-n \omega_{0}\right)^{3} \mid\right\}^{1 / 2}$. In the noninteracting case $g=0$ only the $n=0$ term contributes and the WTD exhibits an initial positive slope for the parameters in Fig. 5, reaching a maximum peak located at $t \approx \pi /|V / 2-\tilde{\epsilon}|$. Increasing the coupling strength, the initial slope decreases and becomes eventually negative. For $g \approx 1.3$, the peak becomes a dip indicating the transition into the strong-coupling regime. The probabilities $P_{1}(t)$ and $P_{2}(t)$ shown in the insets allow us to visualize the injection of the first and second electrons, and their slowing down with increasing interaction.

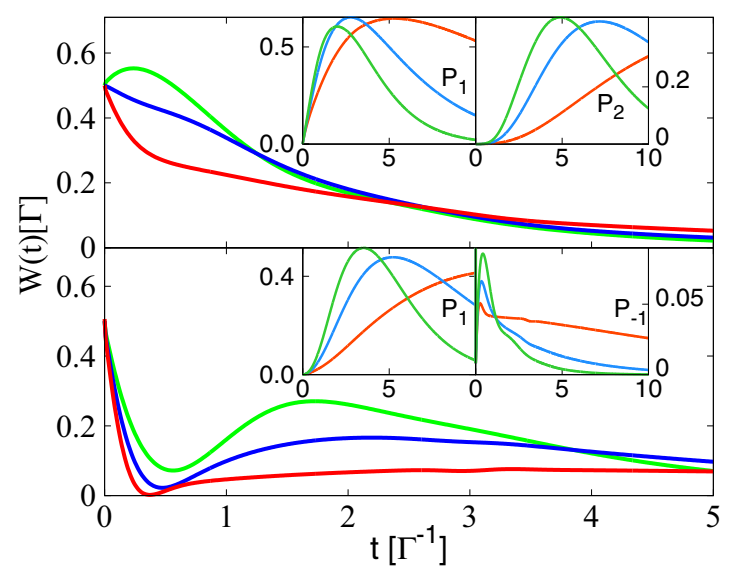

FIG. 5. (Color online) Waiting time distribution for increasing $g$ [0 (green), 1 (blue), and 1.5 (red)] for initially empty (upper panel) and initially full (lower panel) with $\tilde{\epsilon}=-\Gamma, \omega_{0}=2 \Gamma$, and $V=5 \Gamma$. The insets show the corresponding probabilities $P_{1}(t)$ and $P_{2}(t)$ (upper panel) and $P_{1}(t)$ and $P_{-1}(t)$ (lower panel). 

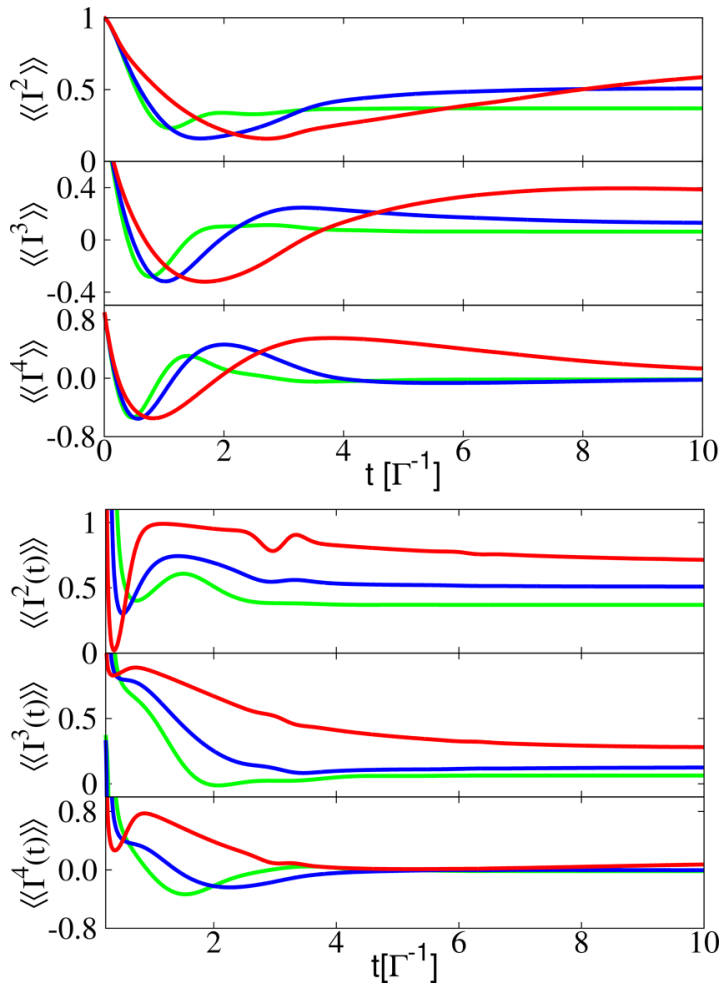

FIG. 6. (Color online) Higher order cumulants (normalized to the mean current) for increasing $g$ values (same color code as in Fig. 5) for the initially empty (upper panels) and initially full cases (lower panels). Notice that in the lower panels the time axis starts at times slightly larger than 0 in order to avoid the divergent behavior of the normalized cumulants in the $t \rightarrow 0$ limit.

On the other hand, the initially occupied case exhibits a different short-time linear scaling for the WTD $W(t) \approx$ $\Gamma_{L} / 2-2 \Gamma_{L} / \pi \sum_{n} \alpha_{n}\left(V / 2-\tilde{\epsilon}+n \omega_{0}\right) t$ followed by a dip at very short times $t \approx \pi\left\{\mid \sum_{n} \alpha_{n}\left(\mu_{L}-\tilde{\epsilon}+n \omega_{0}\right) / \sum_{n} \alpha_{n}\left(\mu_{L}-\right.\right.$ $\left.\left.\tilde{\epsilon}+n \omega_{0}\right)^{3} \mid\right\}^{1 / 2}$ which is associated with the blocking effect of the occupied level. Contrary to the empty case, the evolution of the dip is monotonic with the interaction strength: the dip depth increases and its position shifts towards smaller times with increasing $g$. As shown by the insets, the backward flow probability $P_{-1}(t)$ is quite significant in this case, contrary to the initially empty case.

Similarly, the increase of $g$ has an impact in the evolution and the stationary limit of the higher order cumulants $\left\langle\left\langle I^{k}\right\rangle\right\rangle$. These are shown in Fig. 6 for $k=2,3$, and 4. The cumulants are normalized to the time-dependent mean current, which allows to appreciate more clearly the differences with increasing $g$. As a general feature, both the interacting and noninteracting cases exhibit an increase in the transient amplitude with increasing cumulant order, as already mentioned in connection with Fig. 4. The effect of increasing $g$ is twofold: first, it slows down the dynamics and second, the relative asymptotic values of the cumulants are larger than the noninteracting ones. In the initially occupied case (lower panels in Fig. 6) the same effects can be observed. The divergent relative amplitudes at very short times are due to the change of sign of the mean current occurring in this case.

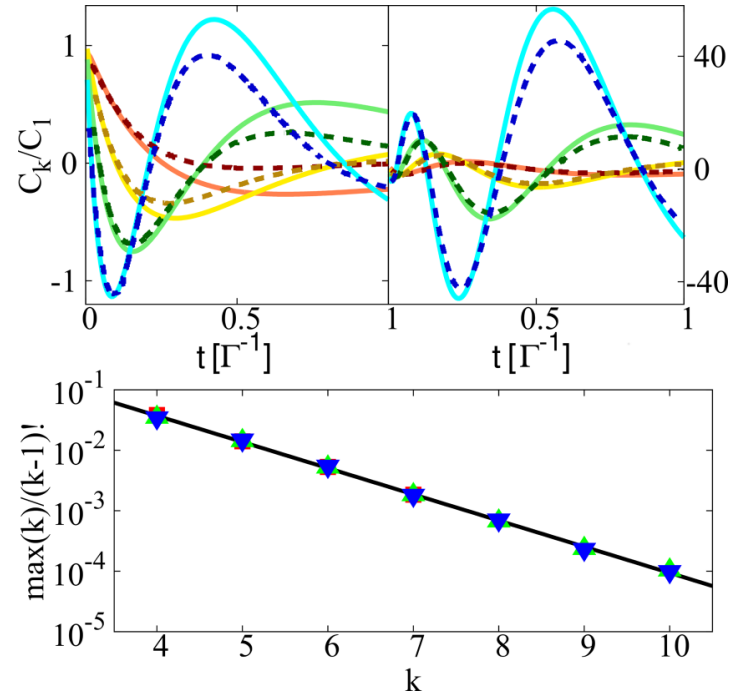

FIG. 7. (Color online) Upper panels: normalized transient current cumulants obtained from Eq. (27) (full lines) and the corresponding results adapted from Ref. [43] (dashed lines) for $k=4,5,6,7$ (left panel) and $k=8,9,10,11$ (right panel). For the comparison we have assumed that $C_{1}(t)$ follows a simple law $A\left(1-e^{-\Gamma_{L} t}\right)$, which sets the time scale. Lower panel: scaling of the maximum amplitude of the relative cumulants $C_{k} / C_{1}$ [indicated by $\max (k)$ in the figure] as a function of $k$. The relative cumulants are normalized with $(k-1)$ ! and the linear slope in the logarithmic scale indicates scaling as $(k-1) ! \pi^{-k}$ (see text). The different symbols correspond to the cases $g=0,1$, and 1.5 in Fig. 5.

\section{Universal scaling of normalized transient cumulants}

In spite of the renormalization of the characteristic times and of the asymptotic cumulants introduced by interactions, for the initially empty case some "universal" features can be identified. For instance, as shown in the lower panel of Fig. 7, the maximum amplitude of the normalized transient cumulants $C_{k}(t) / C_{1}(t)$ are found to follow a scaling which is independent of the value of the interaction parameter $g$. This scaling is also found to be extremely robust with other model parameters such as $\tilde{\epsilon}$ or $\omega_{0}$.

We can explain this behavior by considering the short-time limit discussed in Sec. IIC. For the initially empty case $g_{0}^{+-}\left(t, t^{\prime}\right)=0$ and as Eq. (20) indicates, the current flow is unidirectional. Moreover, in this limit all derivatives of the GF are equal, i.e., $\left.(-i)^{n} \partial^{n} Z(t, \chi) / \partial \chi^{n}\right\rfloor_{0} \equiv x(t)$, corresponding to the situation where just a single electron is involved, as shown in the inset of Fig. 4. Correspondingly, the CGF at short times can be written as

$$
S(\chi, t) \simeq \ln \left[1+x(t)\left(e^{i \chi}-1\right)\right] .
$$

A simple Taylor expansion allows to identify the charge cumulants as

$$
C_{k}(t)=-\sum_{q=1}^{\infty}[-u(t)]^{q} q^{k-1}
$$

where $u(t)=x(t) /[1-x(t)]$. Taking the continuous limit and evaluating the above summation as an integral (valid at 
sufficiently large $k$ ) we find

$$
C_{k}=-\frac{2(k-1) ! \cos \left[k \arctan \left(\frac{\pi}{\ln u}\right)\right]\left(\sigma_{u}\right)^{k}}{\left(\ln ^{2} u+\pi^{2}\right)^{k / 2}},
$$

where $\sigma_{u}=-\operatorname{sgn}(\ln u)$.

While the cosine factor in this expression is responsible for the oscillatory behavior of the cumulants at short times, their maximum amplitude is controlled by the $(k-1)$ ! factor and the power law in the denominator. As in the region of maximum amplitude typically $\ln ^{2} u \ll \pi^{2}$, it is straightforward to show from Eq. (27) that the cumulants' maximum amplitude scales as $(k-1) ! \pi^{-k}$. This law describes with accuracy the scaling of the relative current cumulants already shown in the lower inset of Fig. 7.

The factorial increase of the transient cumulants has been already pointed out and demonstrated experimentally in Ref. [43]. In contrast to the present study, they considered the sequential tunneling regime. However, as we show in the upper panels of Fig. 7, the short-time behavior of the transient cumulants predicted by Eq. (27) remarkably agrees with the results of Ref. [43]. The reason for this agreement is the universality of the generating function in the short-time regime for unidirectional transport corresponding to the initially empty dot case. At longer times the results from Ref. [43] deviate from the predicted behavior by Eq. (27) by a global extra exponential decay, which can be associated with the differences with the setup considered in Ref. [43].

\section{Conductance and Fano factor dynamics at $V=n \omega_{0}$}

It is also worth analyzing the current and noise dynamics for bias voltages close to the conditions $V \sim n \omega_{0}$. The behavior of the stationary noise at the inelastic threshold $V=\omega_{0}$ has been analyzed in several works in the limit of weak interaction [44-47], showing that it can either exhibit an increase or a decrease due to the opening of the inelastic channel. In contrast, for $V=2 \omega_{0}$, the analysis of stationary noise in the polaronic regime presented in Ref. [35] indicates that it exhibits a suppression associated with the opening of a sideband (new elastic channel). The transient conductance for $V \sim \omega_{0}$ and $V \sim 2 \omega_{0}$ is shown in Figs. 8 and 9 for $\tilde{\epsilon}=0, g=1.5$, and different values of $\Gamma$. For comparison we show the prediction for the tunnel limit, given by Eq. (24) as dashed lines. To illustrate the behavior of the noise we choose to represent the differential Fano factor $\partial F(t) / \partial V$, where $F(t)=\left\langle\left\langle I^{2}\right\rangle\right\rangle /(2\langle\langle I\rangle\rangle)$, which allows to see more clearly the differences in the $\Gamma \rightarrow 0$ limit.

As can be observed in these plots, the behavior of the transient conductance for $\Gamma \rightarrow 0$ is remarkably different in the two cases: while for $V=\omega_{0}$ the conductance exhibits a sequence of up and down steps at $t \sim 2 n \pi / \omega_{0}$, for $V=2 \omega_{0}$ the sequence corresponds to steps up only. The approximate behavior is well captured by the analytical expression of Eq. (24) shown as dashed lines in Figs. 8 and 9. When $\Gamma$ increases the step structure in the conductance is progressively damped. On the other hand, the noise exhibits an interesting different evolution with increasing $\Gamma$. While for $\Gamma \rightarrow 0$ the differential noise and the conductance are approximately equal (as expected for the tunnel limit) for larger $\Gamma$ the differential

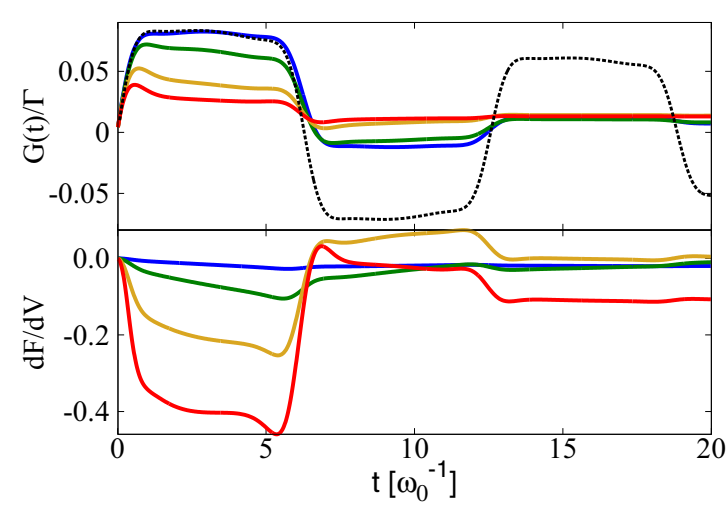

FIG. 8. (Color online) Transient conductance (upper panel) and differential Fano factor (lower panel) for $V=\omega_{0}, g=1.5, \tilde{\epsilon}=0$ and different values of $\Gamma / \omega_{0}[0.05$ (blue), 0.25 (green), 1 (yellow), and 2 (red)]. The dashed line in the upper panel corresponds to the tunnel limit analytical result of Eq. (24).

Fano factor converges to either positive or negative values for $V=\omega_{0}$, or systematically to negative values for $V=2 \omega_{0}$. This is consistent with the predictions of Ref. [35] for the stationary case. It is interesting to remark on the difference between the present calculations and those of Ref. [41]. In that work a sequential tunneling approach was used, including the effect of the induced nonequilibrium phonon population leading to giant Fano factors associated with the Frank-Condon blockade effect.

Finally, we notice that the conductance and differential Fano factor for the case of noncommensurate values of $V$ and $\omega_{0}$ exhibit a less regular evolution, as illustrated in the Supplemental Material [42].

\section{CONCLUSIONS AND OUTLOOK}

In this work we have presented an analysis of the timedependent statistics of electron transport through a resonant level coupled to a localized vibrational mode. We have restricted our analysis to the transient regime which is established after a sudden connection of the level to the leads. For this analysis we have adapted the recently developed DTA decoupling scheme [35], which provides a good description of the stationary transport properties in the polaronic regime.

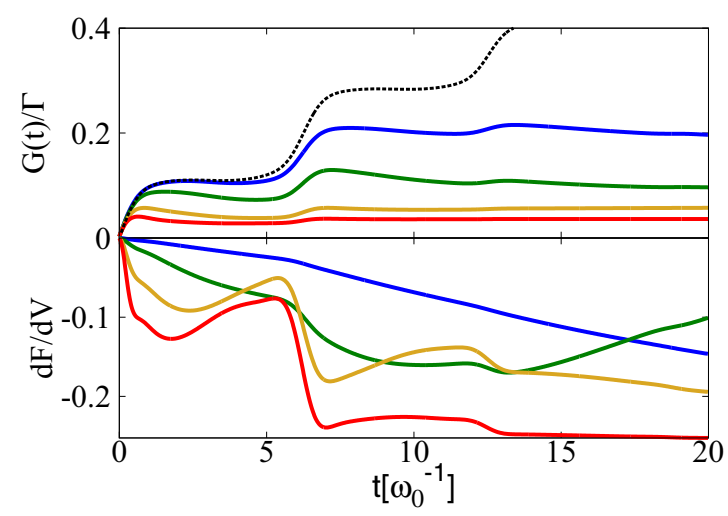

FIG. 9. (Color online) Same as Fig. 8 for $V=2 \omega_{0}$. 
In spite of its approximate character, our analysis has revealed several features of general validity. In the first place it shows that interactions tend to increase exponentially the relevant time scales for the transient dynamics, leading to an apparent bistability at short times in certain parameter regimes, in agreement with a previous analysis by some of us [29]. Second, we have demonstrated that in the short time scale and for an initially empty dot the higher order cumulants exhibit oscillations with a universal scaling amplitude. This universal character arises due to the fact that a single electron transfer controls the transport properties at these short time scales. Our analysis has furthermore revealed a peculiar oscillatory convergence of the conductance and the Fano factor at the inelastic threshold $V=\omega_{0}$.

The present work constitutes a first step in the study of time-dependent statistics for the interacting quantum coherent transport regime. We envisage several possible extensions of this work, such as the study of waiting time distributions in the stationary regime for interacting systems, extending the study to superconducting systems, and analyzing the effect of unequilibrated phonons. This last goal could be achieved by extending previous self-consistent Green's function approaches, such as the one of Ref. [48], to the time domain.

\section{ACKNOWLEDGMENTS}

R.S., R.C.M., A.M.R., and A.L.Y. acknowledge funding from Spanish MINECO through grants FIS2011-26516 and FIS2014-55486-P. R.A. acknowledges funding from French Agence Nationale de la Recherche ANR ORGAVOLT and Partenariats Hubert Curien NANO ESPAGNE Project No. 31404NA.

\section{APPENDIX A: TIME DISCRETIZATION PROCEDURE}

We describe in this appendix the time discretization procedure. For simplicity we discuss here first the noninteracting case. For describing the leads we consider the simplest case of a flat density of states (wide-band approximation) within an energy range in the interval $(-W, W)$. The leads' bandwidth $W$ is assumed to be much larger than the tunneling rates $\Gamma_{v}$. The discretized time-dependent self-energies correspond to the Fourier transform of these energy-dependent self-energies evaluated at the discrete time mesh defined by $t_{j}=\Delta t j$, which at zero temperature yields

$$
\begin{aligned}
& \tilde{\Sigma}_{i j}^{+-}=-\sum_{\nu \equiv L, R} \frac{\Delta t \Gamma_{\nu} e^{i \chi_{\nu}}}{\pi} \frac{e^{-i \mu_{\nu} \Delta t(i-j)}-e^{-i W \Delta t(i-j)}}{(i-j)}, \\
& \tilde{\Sigma}_{i j}^{-+}=-\sum_{\nu \equiv L, R} \frac{\Delta t \Gamma_{\nu} e^{-i \chi_{\nu}}}{\pi} \frac{e^{-i W \Delta t(i-j)}-e^{-i \mu_{\nu} \Delta t(i-j)}}{(i-j)},
\end{aligned}
$$

where $i, j \geqslant 1$. The other self-energy components are given by $\Sigma_{i j}^{++}=-\theta[i-j] \Sigma_{i j}^{-+}-\theta[j-i] \Sigma_{i j}^{+-}$and $\Sigma_{i j}^{--}=$ $-\theta[i-j] \Sigma_{i j}^{+-}-\theta[j-i] \Sigma_{i j}^{-+}$, where $\theta[n]$ is the discrete form of the Heaviside function. These last expressions are not unambiguously defined for $i=j$. We have found that the more stable algorithm corresponds to the choice $\theta[0]=1 / 2$.
The finite flat bandwidth model at zero temperature produces current cumulants increasing linearly from zero, followed by an oscillatory pattern on the time scale $1 / W$, produced by the exponential term in Eq. (A1). This pattern is absent in the symmetrized current cumulants. In order to resolve these features, the time step has to be smaller than this typical time, which leads to the condition of $\Delta t \lesssim 1 / W$ for a stable algorithm. In order to avoid features associated with the finite bandwidth it is convenient to generalize the calculation to finite temperatures, performing an expansion of the self-energies in Matsubara frequencies. In this case, the limit $W \rightarrow \infty$ is well defined, avoiding divergences at time equal to zero. The expressions for the self-energies at finite temperature are

$$
\begin{aligned}
& \tilde{\Sigma}_{j k}^{+-}=2 i \sum_{\nu=L, R} e^{i \chi_{\nu}} \Gamma_{\nu} f_{j k}^{\nu}, \\
& \tilde{\Sigma}_{j k}^{-+}=2 i \sum_{\nu=L, R} e^{-i \chi_{\nu}} \Gamma_{\nu}\left(f_{j k}^{\nu}-\delta[j-k]\right),
\end{aligned}
$$

where the Fermi function can be computed as

$$
\begin{aligned}
f_{j k}^{v}= & i \sum_{n=0}^{\infty} R_{n}\left[\theta[j-k] e^{\beta_{n}(j-k) \Delta t}\right. \\
& \left.-\theta[k-j] e^{-\beta_{n}(j-k) \Delta t}\right] e^{-i \mu_{x}(j-k) \Delta t}+\frac{\delta[j-k]}{2} .
\end{aligned}
$$

Here $\beta_{n}$ and $R_{n}$ represent the poles and the residues of the Matsubara expansion. The convergence speed can be improved by using the approximated poles and residues proposed by Ozaki [49] and computed using a continued fraction. Differently from the zero-temperature finite-bandwidth case, the fast oscillatory term is absent, and the maximum of the time step is controlled by the $\Gamma$ parameter. We have found that $\Delta t \lesssim 1 /(10 \Gamma)$ is sufficient to warrant the convergence of the numerical algorithm.

In the interacting case, the DTA self-energy, given by Eq. (18), can be written as an infinite sum over polaronic weights (see Appendix B). The number of terms needed to be added for convergence is higher when increasing the electron-phonon coupling, meaning that higher sidebands become more important. Considering that the series can be truncated with precision enough at a given integer value, $n$, the condition to converge the calculation can be obtained following the same reasoning done in the noninteracting case; i.e., the polaronic terms generate oscillations of a minimum period $1 /\left(n \omega_{0}\right)$, which have to be resolved for convergence, leading to the condition $\Delta t \lesssim 1 /\left(n \omega_{0}\right)$.

\section{APPENDIX B: DRESSED TUNNELING APPROXIMATION}

In this Appendix we summarize the main expressions of the dressed tunneling approximation (DTA) used in this work. The DTA approximation is built from the Dyson equation, where the leads' self-energies have been dressed with the phonon cloud [35]

$$
\hat{G}=\hat{g}_{0}+\hat{g}_{0} \hat{\Sigma}_{\mathrm{DTA}} \hat{G}
$$

where $\hat{g}_{0}$ corresponds to the undressed dot, $\hat{\Sigma}_{\text {DTA }}$ is the DTA self-energy [as defined in Eq. (18)], and the ${ }^{\wedge}$ symbol is used to 
denote Keldysh structure. In this expression integration over internal times is implicitly assumed. Finally, dressing again the full Green's function, we find the final expression as

$$
\hat{G}_{\mathrm{DTA}}^{\alpha \beta}\left(t, t^{\prime}\right)=\hat{G}^{\alpha \beta}\left(t, t^{\prime}\right) \Lambda^{\alpha \beta}\left(t, t^{\prime}\right),
$$

where the phonon cloud propagator $\Lambda^{\alpha \beta}\left(t, t^{\prime}\right)$ is evaluated assuming equilibrated phonons, i.e.,

$$
\Lambda^{+-}\left(t, t^{\prime}\right)=\left[\Lambda^{-+}\left(t, t^{\prime}\right)\right]^{*}=\sum_{n=-\infty}^{\infty} \alpha_{n} e^{i n \omega_{0}\left(t-t^{\prime}\right)},
$$

with

$$
\alpha_{n}=e^{-g^{2}\left(2 n_{p}+1\right)} I_{n}\left(2 g^{2} \sqrt{n_{p}\left(1+n_{p}\right)}\right) e^{n \beta \omega_{0} / 2},
$$

$I_{n}$ being the modified Bessel function of the first kind, which is symmetric in the $k$ argument $\left(I_{n}=I_{-n}\right)$ and $n_{p}$ is the Bose factor $1 /\left(e^{\beta \omega_{0}}-1\right)$ with $\beta=1 / T$. The remaining components $\Lambda^{++}\left(t, t^{\prime}\right)$ and $\Lambda^{--}\left(t, t^{\prime}\right)$ are determined by $\Lambda^{++}\left(t, t^{\prime}\right)=\theta\left(t-t^{\prime}\right) \Lambda^{-+}\left(t, t^{\prime}\right)+\theta\left(t^{\prime}-t\right) \Lambda^{+-}\left(t, t^{\prime}\right)$ and $\Lambda^{--}\left(t, t^{\prime}\right)=\theta\left(t^{\prime}-t\right) \Lambda^{-+}\left(t, t^{\prime}\right)+\theta\left(t-t^{\prime}\right) \Lambda^{+-}\left(t, t^{\prime}\right)$. In all the calculations presented in this work we have introduced a small temperature of the order of $0.1 \Gamma$ which helps to stabilize the numerical calculations but does not produce significant deviations from the zero-temperature results.

\section{APPENDIX C: SINGLE-POLE APPROXIMATION}

In this Appendix we discuss an approximation that can be used in order to compute the average current and population of the level more efficiently. This approximation is useful in the regime where the electron-phonon coupling is strong and the evaluation of the Fredholm determinant (11) becomes computationally more demanding. For the evaluation of the current and dot population the counting field is not needed and the transformation to the triangular representation in the Keldysh formalism can be performed [50]. Then, the Dyson equation for the retarded component of the Green's function is given simply by

$$
G^{R}\left(t, t^{\prime}\right)=g_{0}^{R}\left(t, t^{\prime}\right)+\int_{0}^{t} d t_{2} K^{R}\left(t, t_{2}\right) G^{R}\left(t_{2}, t^{\prime}\right),
$$

where $K\left(t-t_{2}\right)=\int d t_{1} g_{0}\left(t-t_{1}\right) \Sigma\left(t_{1}-t_{2}\right)$ is the kernel of the integral equation. This kernel is time translationally invariant, meaning that it only depends on the difference between time arguments, which implies that the retarded Green's function, solution to the equation, preserves this symmetry. In this special case, Eq. (C1) can be Laplace transformed arriving at

$$
G^{R}(s)=\frac{g_{0}^{R}(s)}{1-K^{R}(s)} .
$$

The issue of inverting the Laplace transform of a given function is in general not simple, because it usually involves the integration in a Bromwich contour of the function with a given collection of poles. However, if $\Gamma \ll \omega_{0}$, the function has only a single dominant pole, $r$, and the retarded Green's function of the dot can be written simply as

$$
G^{R}\left(t-t^{\prime}\right) \approx \theta\left(t-t^{\prime}\right) \operatorname{Res}\left(G^{R}, s=r\right) e^{r\left(t-t^{\prime}\right)},
$$

where Res indicates the residue at $s=r$. This function is exponentially decaying, with a decay rate given by the real part of $r$.

For the calculation of the current and dot charge evolution we need the $G^{+-}$component, which we analyze below for the initially empty case. For the initially occupied case, similar expressions can be derived by only changing the Green's function component to the $G^{-+}$one. The $G^{+-}$component can be computed using the kinetic equation

$$
G_{\mathrm{DTA}}^{+-}\left(t, t^{\prime}\right)=\int_{0}^{t} d t_{1} d t_{2} G^{R}\left(t, t_{1}\right) \Sigma_{\mathrm{DTA}}^{+-}\left(t_{1}, t_{2}\right) G^{A}\left(t_{2}, t^{\prime}\right),
$$

where the $\Sigma_{\text {DTA }}$ is the self-energy in our DTA approximation. The evolution of the average charge is given by the imaginary part of this Green's function, i.e., $n_{d}(t)=\operatorname{Im}\left[G_{\mathrm{DTA}}^{+-}(t, t)\right]$.

Finally, when the coupling strengths to both electrodes are equal $\left(\Gamma_{L}=\Gamma_{R}\right)$, the symmetrized current can be computed as

$$
\begin{aligned}
\langle I(t)\rangle= & \frac{\theta(t)}{2} \operatorname{Re}\left\{\int_{0}^{t} d t_{1} G_{\mathrm{DTA}}^{R}\left(t, t_{1}\right)\right. \\
& \left.\times\left[\Gamma_{L} f_{L}\left(t-t_{1}\right)-\Gamma_{R} f_{R}\left(t-t_{1}\right)\right]\right\},
\end{aligned}
$$

where

$$
\begin{aligned}
G_{\mathrm{DTA}}^{R}\left(t, t_{1}\right)= & \left\{\Lambda^{+-}\left(t, t_{1}\right) G^{R}\left(t, t_{1}\right)\right. \\
& \left.+\left[\Lambda^{+-}\left(t, t_{1}\right)-\Lambda^{-+}\left(t, t_{1}\right)\right] G_{\mathrm{DTA}}^{+-}\left(t, t_{1}\right)\right\} .
\end{aligned}
$$

[1] Y. M. Blanter and M. Büttiker, Phys. Rep. 336, 1 (2000).

[2] Quantum Noise in Mesoscopic Physics, edited by Y. V. Nazarov (Kluwer, Dordrecht, 2003)

[3] G. Fève, A. Mahé, J.-M. Berroir, T. Kontos, B. Plaçais, D. C. Glattli, A. Cavanna, B. Etienne, and Y. Jin, Science 316, 1169 (2007).

[4] E. Bocquillon, V. Freulon, J.-M. Berroir, P. Degiovanni, B. Plaais, A. Cavanna, Y. Jin, and G. Fève, Science 339, 1054 (2013).

[5] J. Dubois, T. Jullien, P. Roulleau, F. Portier, P. Roche, A. Cavanna, Y. Jin, W. Wegschneider, and D. C. Glattli, Nature (London) 502, 659 (2013).
[6] K. Thibault, J. Gabelli, Ch. Lupien, and B. Reulet, Phys. Rev. Lett. 114, 236604 (2015).

[7] I. Neder and F. Marquardt, New J. Phys. 9, 112 (2007).

[8] N. G. van Kampen, Stochastic Processes in Physics and Chemistry, 3rd ed. (Elsevier, 2007).

[9] T. Brandes, Ann. Phys. (Berlin) 17, 477 (2008).

[10] M. Albert, C. Flindt, and M. Büttiker, Phys. Rev. Lett. 107, 086805 (2011).

[11] L. Rajabi, C. Pöltl, and M. Governale, Phys. Rev. Lett. 111, 067002 (2013).

[12] K. H. Thomas and C. Flindt, Phys. Rev. B 87, 121405(R) (2013).

[13] M. Albert, G. Haack, C. Flindt, and M. Büttiker, Phys. Rev. Lett. 108, 186806 (2012). 
[14] K. H. Thomas and C. Flindt, Phys. Rev. B 89, 245420 (2014).

[15] M. Esposito, U. Harbola, and S. Mukamel, Rev. Mod. Phys. 81, 1665 (2009).

[16] G.-M. Tang, F. Xu, and J. Wang, Phys. Rev. B 89, 205310 (2014).

[17] G.-M. Tang and J. Wang, Phys. Rev. B 90, 195422 (2014).

[18] B. J. LeRoy, S. G. Lemay, J. Kong, and C. Dekker, Nature (London) 432, 371 (2004).

[19] S. Sapmaz, P. Jarillo-Herrero, Y. M. Blanter, C. Dekker, and H. S. J. van der Zant, Phys. Rev. Lett. 96, 026801 (2006).

[20] R. Leturcq, C. Stampfer, K. Inderbitzin, L. Durrer, C. Hierold, E. Mariani, F. von Oppen, and K. Ensslin, Nat. Phys. 5, 327 (2009).

[21] M. Galperin, A. Nitzan, and M. A. Ratner, Phys. Rev. B 74, 075326 (2006).

[22] M. Galperin, M. A. Ratner, and A. Nitzan, J. Phys.: Condens. Matter 19, 103201 (2007).

[23] R. C. Monreal, F. Flores, and A. Martín-Rodero, Phys. Rev. B 82, 235412 (2010).

[24] S. Maier, T. L. Schmidt, and A. Komnik, Phys. Rev. B 83, 085401 (2011).

[25] B. Dong, G. H. Ding, and X. L. Lei, Phys. Rev. B 88, 075414 (2013).

[26] K. F. Albrecht, A. Martin-Rodero, J. Schachenmayer, and L. Mühlbacher, Phys. Rev. B 91, 064305 (2015).

[27] A-P. Jauho, N. S. Wingreen, and Y. Meir, Phys. Rev. B 50, 5528 (1994).

[28] K. F. Albrecht, H. Wang, L. Mühlbacher, M. Thoss, and A. Komnik, Phys. Rev. B 86, 081412 (2012).

[29] K. F. Albrecht, A. Martín-Rodero, R. C. Monreal, L. Muhlbacher and A. Levy Yeyati, Phys. Rev. B 87, 085127 (2013).

[30] E. Perfetto and G. Stefanucci, Phys. Rev. B 88, 245437 (2013); J. Comput. Electron. 14, 352 (2015).

[31] E. Y. Wilner, H. Wang, M. Thoss, and E. Rabani, Phys. Rev. B 89, 205129 (2014).

[32] L. Mühlbacher and E. Rabani, Phys. Rev. Lett. 100, 176403 (2008).
[33] R. Hützen, S. Weiss, M. Thorwart, and R. Egger, Phys. Rev. B 85, 121408 (2012).

[34] A. Jovchev and F. B. Anders, Phys. Rev. B 87, 195112 (2013).

[35] R. Seoane Souto, A. Levy Yeyati, A. Martín-Rodero, and R. C. Monreal, Phys. Rev. B 89, 085412 (2014).

[36] I. G. Lang and Y. A. Firsov, Sov. Phys. JETP 16, 1301 (1962).

[37] G. Mahan, Many-Particle Physics (Plenum Press, New York, 1981).

[38] Y. Utsumi, Phys. Rev. B 75, 035333 (2007).

[39] A. Kamenev, Field Theory of Non-Equilibrium Systems (Cambridge University, Cambridge, 2011).

[40] See, for instance, D. B. Gutman, Y. Gefen, and A. D. Mirlin, J. Phys. A: Math. Theor. 44, 165003 (2011).

[41] J. Koch and F. von Oppen, Phys. Rev. Lett. 94, 206804 (2005).

[42] See Supplemental Material at http://link.aps.org/supplemental/ 10.1103/PhysRevB.92.125435 for a discussion of the relaxation time as a function of the interaction parameter, the range of validity of the universal scaling of the higher order cumulants and the behavior of the conductance and differential Fano factor for the parameters of Figs. 5 and 6.

[43] C. Flindt, C. Fricke, F. Hohls, T. Novotný, K. Netocny, T. Brandes, and R. J. Haug, Proc. Natl. Acad. Sci. USA 106, 10116 (2009).

[44] R. Avriller and A. Levy Yeyati, Phys. Rev. B 80, 041309 (2009).

[45] T. L. Schmidt and A. Komnik, Phys. Rev. B 80, 041307 (2009).

[46] F. Haupt, T. Novotný, and W. Belzig, Phys. Rev. Lett. 103, 136601 (2009).

[47] M. Kumar, R. Avriller, A. L. Yeyati, and J. M. van Ruitenbeek, Phys. Rev. Lett. 108, 146602 (2012).

[48] M. Galperin, A. Nitzan, and M. A. Ratner, Phys. Rev. B 73, 045314 (2006).

[49] T. Ozaki, Phys. Rev B 75, 035123 (2007).

[50] L. Keldysh, Zh. Eksp. Teor. Fiz. 47, 1515 (1964) [Sov. Phys. JETP 20, 1018 (1965)]. 